\title{
Synthesis of Ni nanowires via a hydrazine reduction route in aqueous ethanol solutions assisted by external magnetic fields
}

\section{Y. Zhang, J. Wang, L. M. Wei, P. Liu, H. Wei and Y. F. Zhang*}

One-dimensional $\mathrm{Ni}$ nanostructures were synthesized via a hydrazine reduction route under external magnetic fields. The mixture of de-ionized water and ethanol was used as the reaction solvent and hydrazine hydrate as reducing agents. The morphology and properties of Ni nanostructures were characterized by X-ray diffractometer (XRD), scanning electron microscopy (SEM), and vibrating sample magnetometer (VSM). It was found that the magnetic field strength, concentration of $\mathrm{Ni}$ ions, reaction time and temperature as well as $\mathrm{pH}$ values played key roles on formation, microstructures and magnetic properties of $\mathrm{Ni}$ nanowires. The optimal wires have diameter of $\sim 200 \mathrm{~nm}$ and length up to $\sim 200 \mu \mathrm{m}$. And their coercivity is $\sim 260$ Oe, which is much larger than the commercial $\mathrm{Ni}$ powders of 31 Oe. This work presents a simple, low-cost, environment-friendly and large-scale production approach to fabricate one-dimensional magnetic materials. The resulting materials may have potential applications in conductive filters, magnetic sensors and catalytic agents.

Keywords: Ni fiber; Hydrazine reduction; Magnetic field; Magnetic properties

Citation: L. Y. Zhang, J. Wang, L. M. Wei, P. Liu, H. Wei and Y. F. Zhang, "Synthesis of Ni nanowires via a hydrazine reduction route in aqueous ethanol solutions assisted by external magnetic fields", Nano-Micro Lett. 1, 49-52 (2009). doi: 10.5101/nml.v1i1.p49-52

One-dimensional (1D) magnetic materials such as Fe, Co and Ni have attracted much attention due to their unique magnetic, catalytic, and optical properties with promising applications in catalysts, magnetic sensors, high-density magnetic records, spintronics, magnetic memory bits or microwave circuits, etc [1-6]. Since most physical and chemical properties of these materials depend on their size and shape, it therefore becomes a new and interesting research field to precisely control the size and shape of these materials [7]. Accordingly, 1D nickel nanostructures including nanotubes, nanowires, nanorods, nanochains and nanoarrays, as a type of important magnetic materials, have been synthesized [8-12]. The common methods for preparation of magnetic nanowires are based on porous anodic aluminum oxide (AAO) templates [13]. AAO templates have been proved to be a simple and efficient method in controlling crystal growth and size of the products. Other templates such as polyaniline or polycarbonate membranes, diblock copolymer and mesoporous silica have also been used to fabricate nanowires with excellent quality [14-17]. In view of the complexity of multi-step preparation of the templates and the residue separation after production, template- or surfactant-free methods for the nanowire preparation are desirable. The use of magnetic field assistant in assembling nanoparticles to form 1D magnetic nanostructures have been proposed, which has been applied for preparation of $\mathrm{Co}$ or $\mathrm{Fe}$ nanowires. More recently, this method has been used to synthesize $\mathrm{Ni}$ nanowires [18]. Unfortunately, previous researches are mainly focused on the fabrication in organic solvents by hydrothermal or solvothermal methods which has drawbacks in purification or environment-friendly point of view. 
Therefore, it is imperative to develop simple and template-free methods for fabrication 1D magnetic materials with expected advantages such as low cost, friendly environment, high purity and large-scale production prospection.

Preparation of nanostructures in the aqueous phase may be a preferred approach due to its significant advantages: nonflammable and nontoxic, environment friendly, safety and feasible for a large scale production. However, some limitation of the aqueous approach also should be considered. For example, the as-synthesized products obtained at relatively low temperature are often amorphous, and additional treatment protocols are required to achieve good crystalline of the resulting materials. Additionally, the as-synthesized nanometer-sized $\mathrm{Ni}$ particles tend to be oxidized in aqueous solutions.

In this study, we present an approach for fabrication $\mathrm{Ni}$ nanowires with good crystalline in an aqueous phase solution under normal pressure in absence of any inorganic or organic templates. The influences of magnetic field strength, concentration of $\mathrm{Ni}$ ions and $\mathrm{pH}$ values on the formation and morphology of Ni nanowires were investigated. The magnetic properties of $\mathrm{Ni}$ nanostructures with different morphology were also evaluated.

All chemicals were of analytical reagent grade and used without any further purification. In a typical process, nickel chloride $\left(\mathrm{NiCl}_{2} \cdot 6 \mathrm{H}_{2} \mathrm{O}\right)$ was disolved into the $100 \mathrm{~mL}$ mixture of deionized water and ethanol with a volume ratio of 5:4 to form a transparent absinthe-green solution. An appropriate amount of hydrazine hydrate $\left(\mathrm{N}_{2} \mathrm{H}_{4} \cdot \mathrm{H}_{2} \mathrm{O}, 85 \mathrm{wt} \%\right)$ solution as a reducing agent was added until the absinthe-green sedimentation turned to light blueviolet colour. $\mathrm{NaOH}$ solution $(5 \mathrm{~mol} / \mathrm{L})$ was used to adjust the $\mathrm{pH}$ value of the mixture. The solution was then placed in a $60^{\circ} \mathrm{C}$ water bath and the bath was fixed in a magnetic field. The static magnetic field with interval of $30 \mathrm{~cm}$ and magnetic pole area of $1200 \mathrm{~cm}^{2}$ was generated by direct current flow, and the strength can be controlled from $0.005 \mathrm{~T}$ to $0.5 \mathrm{~T}$ by adjusting the current intensity. After about 30 minutes, loose black floccules was formed and floated on the solution surface. The products were filtered and washed repeatedly with distilled water and ethanol by using magnetic field, and then dried at $60^{\circ} \mathrm{C}$ for 12 hours.

The size and morphology analyses were performed using field emission scanning electron microscope (SEM, Ultrazeiss, Zeiss). The phase structure was characterized by X-ray polycrystalline diffractometer (XRD, D8 Advance, Bruker) using $\mathrm{Cu} \mathrm{Ka}$ radation with graphite monochromator. The hysteresis properties were measured on a vibration sample magnetometer (VSM, Lake Shore 7400).

Figure 1 shows the SEM images of resulting products with $\mathrm{Ni}^{+}$concentration of $0.01 \mathrm{M}$ under different applied magnetic fields. It can be seen that the magnetic field has strong influences on the morphology of products. In the absence of the magnetic field, only some bulky particles were formed and no 1D material was observed (see Fig. 1a), whereas, under a low magnetic field of $0.05 \mathrm{~T}$, some short, thick and flexural wires were observed (see Fig. 1b). When the strength of the magnetic field was increased to $0.2 \mathrm{~T}$, the longer and thinner nanowires can be formed and no separated particles were observed. The wires have aspect ratio of about 1000 with average diameter of $200 \mathrm{~nm}$ and length of $200 \mu \mathrm{m}$ (see Fig. 1c). There is little change observed on morphology of nanowires when magnetic fields increased from $0.2 \mathrm{~T}$ to $0.5 \mathrm{~T}$.
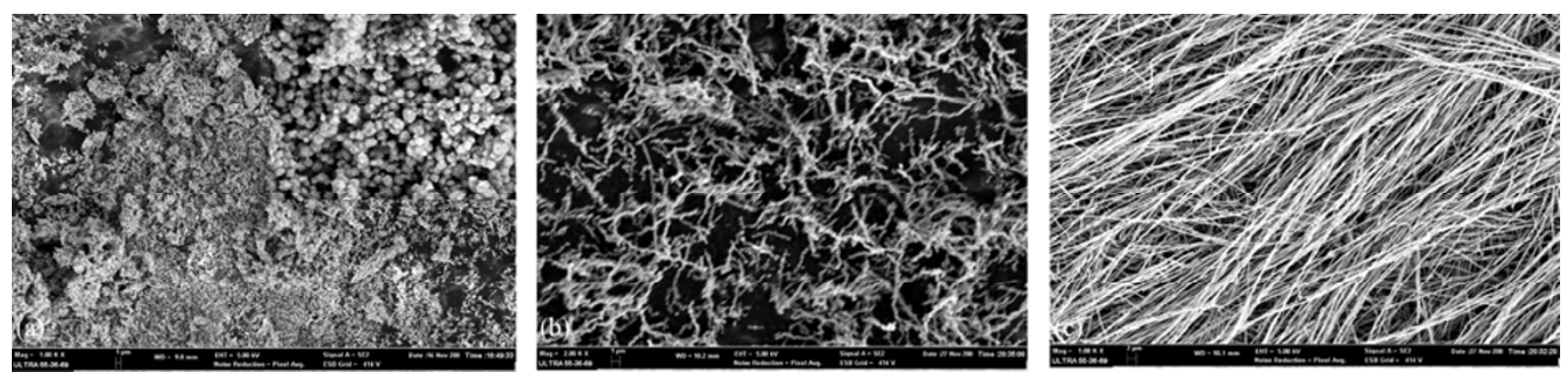

FIG. 1. SEM images of samples prepared at different magnetic fields: (a) 0; (b) $0.05 \mathrm{~T}$; (c) $0.20 \mathrm{~T}$.
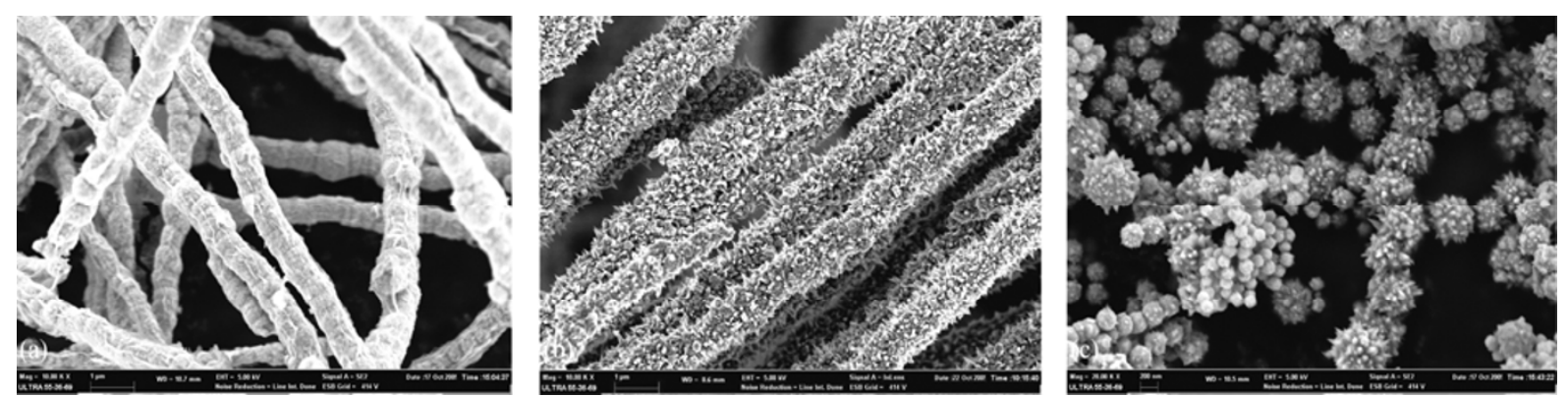

FIG. 2. SEM images of products prepared in different Ni ion concentration under a $0.3 \mathrm{~T}$ magnetic field: (a) $0.01 \mathrm{M}$; (b) $0.05 \mathrm{M}$; (c) $0.1 \mathrm{M}$. 

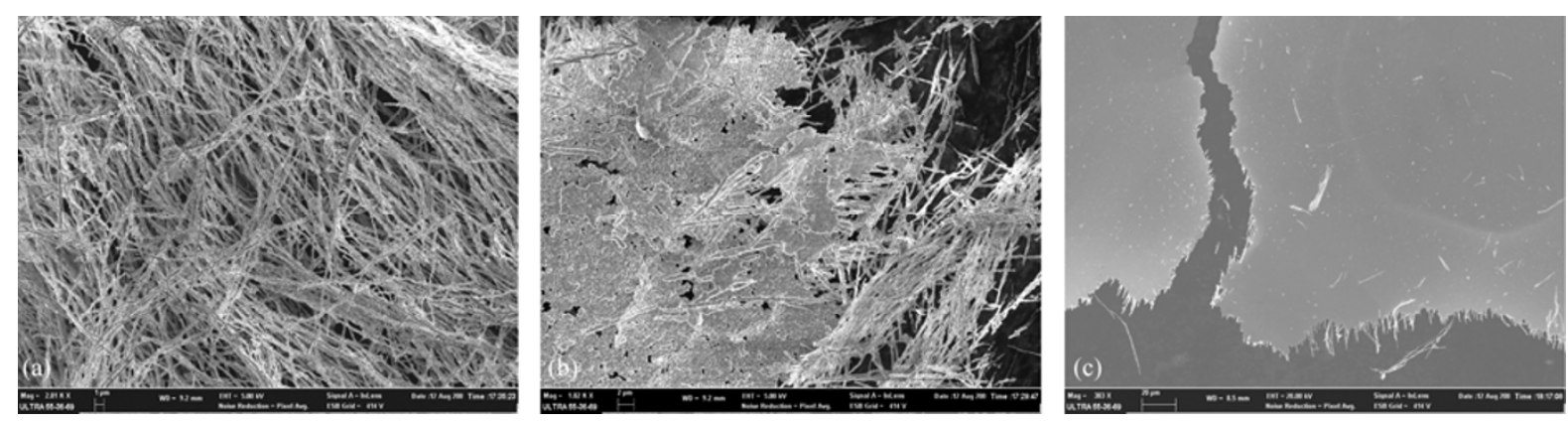

FIG. 3. SEM images of products prepared in different time under a $0.3 \mathrm{~T}$ magnetic field: (a) $0.5 \mathrm{~h}$; (b) $2 \mathrm{~h}$ (c) $3 \mathrm{~h}$.

The influence of concentration of Ni ions on morphology of Ni nanowires has also been observed. Figure 2 shows the SEM images of products with different $\mathrm{Ni}$ ion concentration under the same magnetic field of $0.2 \mathrm{~T}$. It can be seen that the dimeter of the nanowires increased with increasing $\mathrm{Ni}^{+}$ concentration. It is about $200 \mathrm{~nm}$ for concentration of $0.01 \mathrm{M}$, and about $600 \mathrm{~nm}$ for $0.05 \mathrm{M}$. When the concentration increased to $0.1 \mathrm{M}$, there is no wires observed, whereas, the clusters with diameter from 200 to $600 \mathrm{~nm}$ were produced.

The reaction time has strong influence on the morphology of the products. There are no products observed less than 10 min. Some loose black floccules were formed and floated on the solution surface after $10 \mathrm{~min}$ later and for about $30 \mathrm{~min}$ the solution became transparent, clarifying and colorless. All the products were floated on the solution surface. However, as the reaction time elongated to $2 \mathrm{~h}$, the nanowires will join together to form silvery-white flakes. For about $3 \mathrm{~h}$ later, the grey-colored nanowires disappeared gradually, and bright silvery-white films were formed on the beaker wall. The products dependence of reaction time was displayed in Fig. 3.

The XRD pattern of the Ni nanowires prepared at $0.01 \mathrm{M}$ concentration under $0.2 \mathrm{~T}$ magnetic field is shown Fig. 4. Three diffraction peaks corresponding to different crystallization directions of [111], [200] and [220], respectively, indicate the nanowire has a cubic crystal structure which is in agreement well with the Nickel standard card (no. 65-0380). There is no other impurity observed, suggesting the prepared Ni nanowires

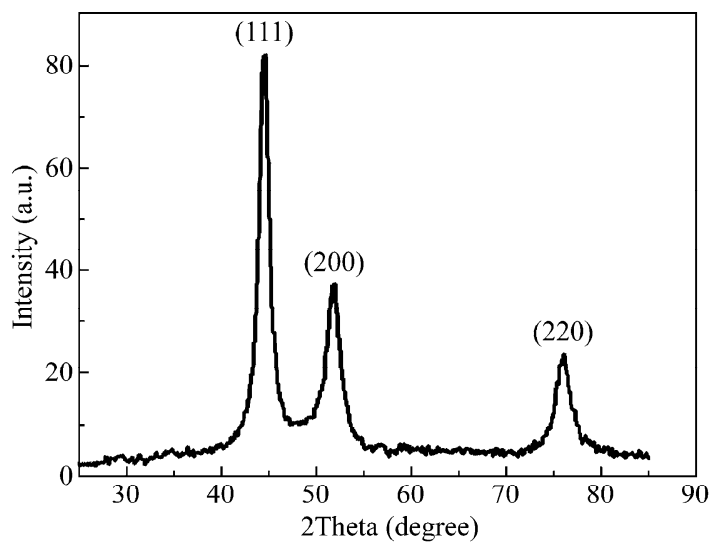

FIG. 4. XRD pattern of Ni nanowires. have high purity.

Figure 5 shows the hysteresis loops of the Ni nanowires which prepared at magnetic field of $0.2 \mathrm{~T}$. The magnetic property of commercial Ni powder which has particle size of about 1 um was also investiged. The nanowires have coercivity of 260 Oe which is much larger than 31 Oe of the powder. However, the saturation magnetization of the $\mathrm{Ni}$ nanowires is $41 \mathrm{emu} / \mathrm{g}$, which is much less than the $55.5 \mathrm{emu} / \mathrm{g}$ of the powder sample. The large coercivity of Ni nanowire attributes to the shape anisotropy due to its large aspect ratio. This phenomenon has been observed on other $\mathrm{Ni}$ nanowires which prepared by templated-assited methods. The reduced magnetization of $\mathrm{Ni}$ nanowires may result from the surface effect of Ni nanowire structures, in which lots of $\mathrm{Ni}$ atoms at the surface are not aligned along its magnetic anisotropy direction in order to reduce the the surface energy. Therefore, the magnetic moments of these $\mathrm{Ni}$ atoms cannot be aligned along the magnetic field due to the strong exchange interaction.

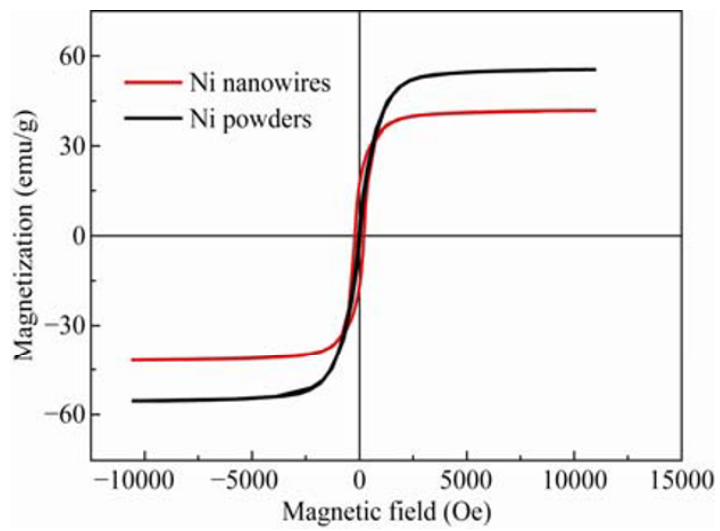

FIG. 5. Hysteresis loops of Ni nanowires and commercial Ni powders.

The possible mechanism for the formation of Ni nanowires in alkali solution under magnetic assistant may be understood as following: nickel ions were firstly reducted by strong reduction agent of hydrazine hydrate and turned to tiny spherical particles. Then the magnetic Ni particles aligned along the magnetic field direction under the magnetic driving force. The nickel nanowires retained their linear structure after kept in ultrasonic bath for 10 minutes, which proved that the nanowires 
showed a good mechanical strength.

It was found that the $\mathrm{pH}$ value of the reaction solution is sensitive to the reduction process of nickel ion by hydrazine hydrate. When the $\mathrm{pH}$ value was higher than 13.70 , the nickel ion can be completely reduced within $30 \mathrm{~min}$, whereas, when the $\mathrm{pH}$ value was adjusted to 13.00 , it will need 2 hours to finish the reaction. If the $\mathrm{pH}$ value was lower than 13.00 , the reaction would not take place and there was no any $\mathrm{Ni}$ nanowires or particles produced. This is attributed to the different oxidation reduction potentials $\varphi_{0}$ of the reaction for different $\mathrm{pH}$ values. For a low $\mathrm{pH}$ condition, the reaction takes place as follows and the reduction potential can be calculated:

$$
\begin{array}{ll}
\mathrm{N}_{2} \mathrm{H}_{5}^{+}-4 \mathrm{e}=5 \mathrm{H}^{+}+\mathrm{N}_{2}, & \varphi_{0}=-0.23 \mathrm{~V} \\
\mathrm{Ni}^{2+}+2 \mathrm{e}=\mathrm{Ni}, & \varphi_{0}=-0.25 \mathrm{~V}
\end{array}
$$

whereas, for a high $\mathrm{pH}$ condition, they will be:

$$
\begin{array}{ll}
\mathrm{Ni}(\mathrm{OH})_{2}+2 \mathrm{e}=\mathrm{Ni}, & \varphi_{0}=-0.72 \mathrm{~V} \\
\mathrm{~N}_{2} \mathrm{H}_{4}+4 \mathrm{OH}^{-}-4 \mathrm{e}=\mathrm{N}_{2}+4 \mathrm{H}_{2} \mathrm{O}, & \varphi_{0}=-1.15 \mathrm{~V}
\end{array}
$$

The oxidation reduction potentials at low $\mathrm{pH}$ condition are higher than those for higher $\mathrm{pH}$ values. This will lead to the reaction much easier for higher $\mathrm{pH}$ conditions.

In this method all the reaction agents are common and low-cost. The reaction process is simple and fast. It provides a new strategy to prepare other magnetic nanowires. In addition, the amount of products can be up to $100 \mathrm{~g}$ /day in our laboratory. So it is also a feasible and potential approach for large scale synthesis of $\mathrm{Ni}$ nanowires. Although the diameter of the nanowires can be tuned by changing magnetic field and the concentration of nickel ion, the diameter less than $200 \mathrm{~nm}$ and more smooth surface nanowires with single crystal structure which synthesized in an ambient aqueous condition is still a challenge. It may be obtained under a much higher magnetic field or to find more suitable experimental conditions. The reaction mechanism should be understood much more. The more detailed research is ongoing.

A simple, low-cost, environment-friendly approach of preparation magnetic $\mathrm{Ni}$ nanowires was developed. In this method, the nanowires were fabricated in an ambient aqueous solution at normal pressure by assistant of magnetic field without any inorganic or organic templates or any other surfactants. The prompt wires have mean diameter of $200 \mathrm{~nm}$ and length up to $200 \mu \mathrm{m}$. It was found that the diameter of wires increases with increasing strength of magnetic field, and there is no wires formed in absence of magnetic field. The $\mathrm{pH}$ value and the concentration of nickel ion has also strong influences on formation and morphology of nanowires. The wires formed only below $0.1 \mathrm{M}$ of $\mathrm{Ni}$ concentration and $\mathrm{pH}$ value should be up to 13 at $60^{\circ} \mathrm{C}$. This method provides a new approach to fabricate magnetic nanowires in an ambient condition and may be the most promising candidate to produce large-scale magnetic nanowires.

The authors gratefully acknowledge the support of the National
Basic Research Program of China (No.2006CB300406); Shanghai Science and Technology Grant (No: 0752nm015), National Natural Science Foundation of China (No. 50730008, 20504021), Natural Science Foundation of Shanghai (No. 09ZR1414800), Shanghai Applied Materials Collaborative Research Program (No: 09520714400).

Received 20 November 2009; accepted 5 December 2009; published online 20 December 2009.

\section{References}

1. W. Zhou and L. Guo, Chem. Bull. 69, w063 (2006).

2. L. Tan, Q. Li, Y. Yang, F. Li, L. Liu and M. Li, Mater. Rev. 17, 41 (2003).

3. L. Tan, Q. Li, Y. Yang, F. Li, L. Liu and M. Li, J. Solid Rocket Technol. 27, 198 (2004).

4. M. A. Ermakova, D. Y. Ermakov, S. V. Cherepanova and L. M. Plyasova, J. Phys. Chem. B 106, 11922 (2002). doi:10.1021/jp021231q

5. J. Bao, C. Tie, Z, Xu, Q. Zhou, D.Shen and Q. Ma, Adv. Mater. 13, 1631 (2001).

6. S. Z. Chu, K. Wada, S. Inoue and S. Todoroki, Chem. Mater. 14, 4595 (2002). doi:10.1021/cm020272w

7. E. K. Athanassiou, P. Grossmann, R. N. Grass and W. Stark, J. Nanotech. 18, 165606 (2007).

8. M. Tanase, L. A. Bauer, A. Hultgren, D. M. Silevitch, L. Sun, D. H. Reich, P. C. Searson and G. J. Meyer, Nano Lett. 1, 155 (2001). doi:10.1021/n1005532s

9. H. Pan, B. Liu, J. Yi, C. Poh, S. Lim, J. Ding, Y. Feng, C. H. A. Huan and J. Lin, J. Phys. Chem. B 109, 3094 (2005). doi:10.1021/jp0451997

10. S. Z. Chu, K. Wada, S. Inoue, S. Todoroki, Y. K. Takahashi and K. Hono, Chem. Mater. 14, 4595 (2002). doi:10.1021/cm020272w.

11. M. Knez, A. M. Bittner, F. Boes, C. Wege, H. Jeske, E. Maib and K. Kern, Nano Lett. 3, 1079 (2003). doi:10.1021/n10342545

12. H. Niu, Q. Chen, M. Ning, Y. Jia and X. Wang, J. Phys. Chem. B 108, 3996 (2004). doi:10.1021/jp0361172

13. J. Qin, J. Nogues, M. Mikhaylova, A. Roig, J. S. Munoz and M. Muhammed, Chem. Mater. 17, 1829 (2005). doi:10.1021/cm047870q

14. H. Q. Cao, L. D. Wang, Y. Qiu, Q. Z. Wu, G. Z. Wang, L. Zhang and X. W. Liu, Chem. Phys. Chem. 7, 1500 (2006). doi:10.1002/cphc.200500690

15. S. H. Ge, C. Li, X. Ma, W. Li, L. Xi and C. X. Li, J. Appl. Phys. 90, 509 (2001). doi:10.1063/1.1327599

16. I. W. Hamley, Nanotechnology 14, R39 (2003).

17. H. M. Luo, D. H. Wang, J. B. He and Y. F. Lu, J. Phys. Chem. B 109, 1919 (2005). doi:10.1021/ip045554t

18. F. L. Jia, L. Z. Zhang, X. Y. Shang and Y. Yang. Adv. Mater. 20, 1050 (2008). doi:10.1002/adma.200702159 